\title{
Effect of DNA charge helicity on $B-Z$ DNA transition
}

\author{
A. G. Cherstvy ${ }^{\text {a) }}$ \\ Institut für Festkörperforschung, Forschungszentrum Jülich, 52425 Jülich, Germany \\ and Max-Planck-Institut für Physik Komplexer Systeme, Nöthnitzer Straße 38, \\ 01187 Dresden, Germany
}

(Received 8 April 2005; accepted 2 June 2005; published online 16 September 2005)

[DOI: $10.1063 / 1.2036990]$

\section{INTRODUCTION}

At standard conditions DNA adopts the right-handed $B$ form with about ten base pairs (bps) per turn. ${ }^{1}$ In solutions, several factors can affect its stability and induce a change in DNA structure from $B$ to $Z$ form (specific ions, temperature, dehydrating solvents, osmotic pressure, $p \mathrm{H}$, etc.). In dense assemblies, DNA structural transitions can also be triggered by DNA-DNA intermolecular interactions $[B-A$ transition in DNA crystals at low humidity ${ }^{2}$ and $B-Z$ transition in Ni(II)induced DNA condensation ${ }^{3}$. The $B-Z$ transition for DNA with alternating $C G$ bps occurs at a high concentration of salt $n_{0}, \sim 2.5 \mathrm{M}$ of $\mathrm{NaCl}^{4-6}$ This happens in a cooperative manner, ${ }^{4}$ reminiscent of DNA melting transition. ${ }^{7,8}$ Experiments have shown that the free-energy difference between $Z$ and $B$-DNA, $E_{Z}-E_{B}$, decreases almost linearly with $\ln \left[n_{0}\right]$, for $n_{0}$ between $1 M$ and $5 M{ }^{5}$ This quantity is the major focus of the present paper. Z-DNA is a left-handed zigzaglike helix, it is thinner than $B$-DNA, its pitch with 12 bps is larger, and the width of the minor groove is smaller than those for $B$ form. ${ }^{9}$ Below, we incorporate these details of molecular structure in our electrostatic model.

Several explanations of $B-Z$ transition exist. The most known one suggests that as the phosphates on $Z$-DNA are closer than on $B$-DNA, the $Z$ form should be more stable at high salt when the repulsion of phosphates is screened better. Another explanation is based on the economy of hydration of DNA phosphates (hydrated better than DNA bases or sugars): at high water activity the phosphates are hydrated individually favoring the $B$ form, whereas with the addition of salt a collective hydration of phosphates can occur. ${ }^{10}$ That induces a bridging of adjacent phosphates by water and changes the conformation from $B$ to $Z$ where phosphates are indeed closer. A better solvation of Z-DNA and higher counterion concentration in its vicinity compared to those of $B$-DNA were also suggested as $Z$-DNA-stabilizing factors. ${ }^{11}$

As DNA is a highly charged molecule, several polyelectrolyte models of the $B-Z$ transition were developed. Some of them have modeled DNAs as uniformly charged rods, ${ }^{12,13}$ and have shown that $Z$-DNA stabilizes at high salt because of its higher surface charge density, as compared to $B$-DNA. ${ }^{13}$ The computed slope of $E_{Z}-E_{B}$ as a function of $n_{0}$ was however an order of magnitude smaller than in experiments. Other electrostatic theories do include fine details of DNA structure, its helical charge motif, different accessibility of DNA surface for ions, ${ }^{14-18}$ as well as the effects of ion-ion and ion-phosphate correlations. ${ }^{19}$ For instance, it has been shown that the slope of the energy difference can depend dramatically on the radius of ions in the solution. ${ }^{19}$ Some of these models give a good agreement with experimental data, but they contain adjustable parameters and questionable assumptions. Also, a superposition of many different effects in these models does not provide a clear physical picture.

\section{ELECTROSTATIC ENERGY}

Below, we treat the effect of DNA helicity on the $B$ to $Z$ transition. We consider only the salt-dependent electrostatic energy term for DNA helices and neglect nonelectrostatic contributions (water hydration, steric correlations, etc.). We use the electrostatic energy of charged spirals for a description of the energetics of the $B-Z$ DNA transition. We model DNA phosphates as two coaxial infinitely thin negatively charged spirals with a proper linear charge density, which are mutually shifted by the distance $d$ along their common axis. This two-spiral model should give a more realistic description of DNA electrostatics than the featureless cylinder used before. ${ }^{12,13}$ We expect that such a model of continuous spirals should catch the effect of DNA helicity. For clarity, we do not consider here the effect of counterions condensed on DNA. ${ }^{20}$

The electrostatic energy $E_{\mathrm{el}}$ and potential of this doublespiral charge distribution has recently been calculated within the linear Poisson-Boltzmann theory. ${ }^{21}$ The linearity of equations has allowed us to treat helicity effects analytically. This calculation has shown that the potential in DNA minor groove is lower that in the major groove because of the closer proximity of charge strands. The energy $E_{\mathrm{el}}$ is the sum of the energy of a uniformly charged $\operatorname{rod} E_{\text {rod }}$ and corrections to it coming from the charge helicity. Per DNA bp one can get $^{21}$

$$
\begin{aligned}
E_{\mathrm{el}}= & \frac{4 \pi^{2} \sigma^{2} a^{2} 2 b}{\varepsilon} \\
& \times\left\{\frac{K_{0}(\kappa a)}{\kappa a K_{1}(\kappa a)}+2 \sum_{n=1}^{\infty} \frac{[1+\cos (2 \pi n d / H)] K_{n}\left(\kappa_{n} a\right)}{\kappa_{n} a\left[K_{n+1}\left(\kappa_{n} a\right)+K_{n-1}\left(\kappa_{n} a\right)\right]}\right\} .
\end{aligned}
$$

Here $\sigma$ is the mean DNA surface charge density (for $B$-DNA $\sigma_{B} \approx 16.6 \mu \mathrm{C} / \mathrm{cm}^{2}$ and for Z-DNA $\sigma_{Z}$ $\left.\approx 20.4 \mu \mathrm{C} / \mathrm{cm}^{2}\right), a$ is the radius of the spiral $\left(a_{B} \approx 9 \AA\right.$, $\left\langle a_{Z}\right\rangle \approx 6.8 \AA$ (Ref. 22), $b$ is the distance between closest unit charges along the DNA axis $\left(b_{B} \approx 1.7 \AA, b_{Z} \approx 1.83 \AA\right), H$ is the DNA helical pitch $\left(H_{B} \approx 34 \AA, H_{Z} \approx 44 \AA\right), d$ is the 


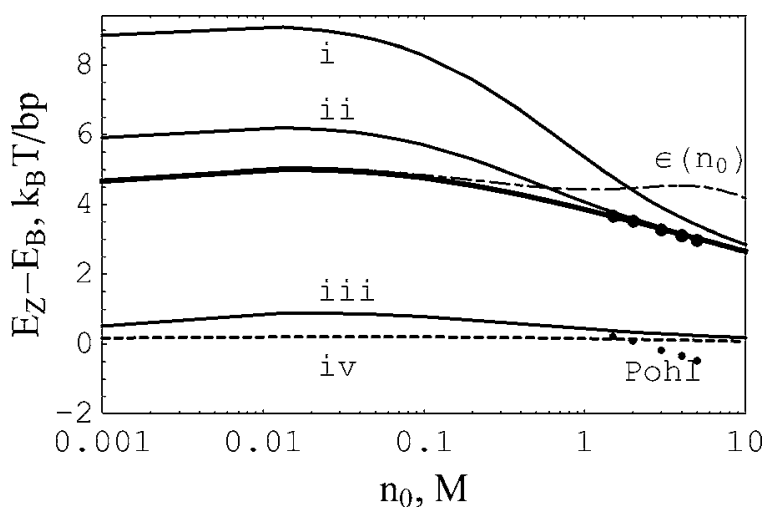

FIG. 1. Electrostatic energy difference of $B$ - and Z-DNA as a function of $n_{0}$ for $d_{Z}=15.3 \AA$ (bold solid), $10 \AA$ (ii) $5 \AA$ (i), and for uniformly charged rods (iii). For the dashed-dotted curve $\varepsilon\left(n_{0}\right)$ dependence (Ref. 25) is taken into account for the energy of the helices. The dots are the data of Pohl (Ref. 5); large dots are the same data but just shifted to improve comparing with our results. Curve (iv) is obtained in Ref. 12 from the nonlinear PoissonBoltzmann theory for uniformly charged DNA rods with $a_{B}=10 \AA$ and $a_{Z}$ $=8.5 \AA$.

height of DNA minor groove $\left(d_{B} \approx 13.6 \AA, d_{Z} \approx 15.4 \AA\right)$, $\kappa_{n}^{-1}=\left[\kappa^{2}+n^{2}(2 \pi / H)^{2}\right]^{-1 / 2}$ are the modified screening lengths which account for the screening in solution with the length $\kappa^{-1}=\left(8 \pi l_{B} n_{0}\right)^{-1 / 2}$ and for helix-related screening, $l_{B}$ $=e_{0}^{2} /\left(\varepsilon k_{B} T\right)$ is the Bjerrum length, $\varepsilon$ is the water dielectric constant, and $K_{n}(x)$ are the modified Bessel functions of $n$th order. $^{23}$

\section{RESULTS AND DISCUSSION}

We plug these structural parameters of $B$ - and Z-DNAs into Eq. (1) and plot in Fig. 1 the difference of their electrostatic energy, together with the experimental data of Pohl. ${ }^{5}$ Our results show that Z-DNA is stabilized at high $n_{0}$ and the slope of $E_{Z}\left(n_{0}\right)-E_{B}\left(n_{0}\right)$ is very close to the experimental one [small dots are taken from Fig. 1(b) in Ref. 5]. We obtain the result that $B$-DNA is more stable at intermediate $n_{0}$, in agreement with Ref. 12. In Fig. 1 we also plot the results for $d_{Z}$ $=10 \AA$ and $5 \AA$ to get a feeling of the effect of the DNAs' groove width. We obtain that the slope of $E_{Z}-E_{B}$ increases as $d_{Z}$ becomes smaller (i.e., when Z-DNA strands become closer across the minor groove). If DNAs were considered as uniformly charged rods ${ }^{12}$ [the first term in Eq. (1)], Z-DNA also gets stabilized at high $n_{0}$ [curve (iii) in Fig. 1]. This term is the same as one gets by the rod charging procedure presented in Ref. 12. However, the effect of salt on such rodlike "DNA" is much weaker than that in experiments: ${ }^{5}$ it is an $\sim 0.05 k_{B} T /$ bp energy decrease for $n_{0}$ which increases from $1 M$ to $5 M .^{13}$ This reveals the crucial role of DNA charge helicity explored in the present paper for description of DNA electrostatic stability at high salt.

The value of $\varepsilon$ in Eq. (1) was assumed to be independent of $n_{0}$. However, for a $\mathrm{NaCl}$ solution $\varepsilon$ goes down considerably for large salt amounts. ${ }^{25}$ If one accounts for this, $E_{\text {rod }}$ increases as compared to the $\varepsilon=80$ situation and the results for $E_{Z}\left(n_{0}\right)-E_{B}\left(n_{0}\right)$ start to disagree with experimental data [Fig. 1]. A reduced stability of Z-DNA with a decrease of $\varepsilon$ was also obtained in computer simulations. ${ }^{14}$ One possible explanation for this is a twofold role of $\varepsilon$. Namely, a de- crease of $\varepsilon$ with $n_{0}$ can affect not only $\kappa$ but also net DNA charge. DNA can become more neutralized by condensed counterions due to their stronger interaction with DNA phosphates (electrostatic interactions grow as $\varepsilon$ goes down).

The present model is applicable basically for nonspecific ion-DNA interactions. However, several cations (e.g., divalent transition metals, ${ }^{27}$ spermine, and cobalt hexammine ${ }^{28}$ ) are known to adsorb specifically into the DNA major groove $^{29}$ and trigger $B-Z$ transition for poly $[\mathrm{d}(\mathrm{G}-\mathrm{C})] .{ }^{27}$ Typical concentrations required for this are in the millimeter range, i.e., much smaller than those required for $\mathrm{NaCl}$ induced high-salt $B-Z$ transition. The driving force for such low-salt transition is likely a better accessibility of the major groove adsorption sites on $Z$-than on $B$-DNA (one of the strongest sites is $\mathrm{N} 7$ of guanine). This mechanism of $B-Z$ transition may also govern sequence-dependent DNA condensation in the presence of transition metal cations. ${ }^{3,34}$

${ }^{a)}$ Electronic mail: cherstvy@mpipks-dresden.mpg.de

${ }^{1}$ R. E. Franklin and R. G. Gosling, Nature (London) 171, 740 (1953); J.

D. Watson and F. H. C. Crick, Nature (London) 171, 737 (1953).

${ }^{2}$ R. Podgornik, H. H. Strey, K. Gawrisch, D. C. Rau, A. Rupprecht, and V. A. Parsegian, Proc. Natl. Acad. Sci. U.S.A. 93, 4261 (1995).

${ }^{3}$ J. C. Sitko et al., Biophys. J. 84, 419 (2003).

${ }^{4}$ F. M. Pohl and T. M. Jovin, J. Mol. Biol. 67, 375 (1972).

${ }^{5}$ F. M. Pohl, Cold Spring Harbor Symp. Quant. Biol. 47, 113 (1983).

${ }^{6}$ These enormous salt amounts may distort that water hydrogen bonds network and thus affect hydrophobic interactions between stacked DNA bps. The type of anions can also influence the $B-Z$ transition: for $\mathrm{NaCl}$ the transition takes place at higher salt than for $\mathrm{NaClO}_{4}$ (chaotropic ions such as perchlorates are known as water structure breakers).

${ }^{7}$ Yu. S. Lasurkin et al., Biopolymers 9, 1253 (1970).

${ }^{8}$ A. G. Cherstvy and A. A. Kornyshev, J. Phys. Chem. B 109, 13024 (2005).

${ }^{9}$ H. J. Wang et al., Nature (London) 282, 680 (1979).

${ }^{10}$ W. Saenger, W. N. Hunter, and O. Kennard, Nature (London) 324, 385 (1986).

${ }^{11}$ V. K. Misra and B. Honig, Biochemistry 35, 1115 (1996).

${ }^{12}$ M. D. Frank-Kamenetskii et al., J. Biomol. Struct. Dyn. 3, 35 (1985); 8, 1113 (1991); 9, 517 (1991).

${ }^{13}$ M. D. Frank-Kamenetskii et al., Sov. Phys. Usp. 30, 317 (1987).

${ }^{14}$ M. Gueron and J. P. Demaret, Proc. Natl. Acad. Sci. U.S.A. 89, 5740 (1992).

${ }^{15}$ J. P. Demaret and M. Gueron, Biophys. J. 65, 1700 (1993).

${ }^{16}$ M. Gueron, J. P. Demaret, and M. Filoche, Biophys. J. 78, 1070 (2000).

${ }^{17}$ J. L. Abascal and J. C. G. Montoro, J. Phys.: Condens. Matter 12, A327 (2000).

${ }^{18}$ It was suggested that as $B$-DNA has deeper grooves, its phosphates are well immersed into the solution, the ions can "enter" the grooves and thus screen better the repulsion of phosphates (Ref. 14). For Z-DNA, the phosphates are closer to the molecular core, the major groove is shallow, and the ions can only surround DNA from outside and thus screening of phosphates is weaker.

${ }^{19}$ D. M. Soumpasis, Proc. Natl. Acad. Sci. U.S.A. 81, 5116 (1984).

${ }^{20}$ Equation (1) does not contain the effect of counterions condensed on DNA molecule. One can, however, account for this as well. For instance, if the charge of adsorbed cations were homogeneously smeared, compensating the fraction $\theta$ of DNA phosphate charge, the first term in Eq. (1) should be multiplied by $(1-\theta)^{2}$ (Ref. 21). As we show below, however, the results of the model depend only weakly on the magnitude of this "homogeneous" term. In reality, the adsorbed counterions build some nonhomogeneous charge pattern on DNA, being adsorbed in the grooves and on the strands. The total amount and partitioning of adsorbed cations is not known precisely. These quantities may not be the same for the two DNA forms considered and can also change with salt concentration. That is, accounting for the effect of condensed counterions would add four poorly known parameters into our model. To avoid this, we neglect here for clarity the condensed cations and stay within our oversimplified but tractable model.

${ }^{21}$ A. G. Cherstvy and R. G. Winkler, J. Chem. Phys. 120, 9394 (2004). 
${ }^{22}$ The phosphates of Z-DNA are positioned on two distinct separations (about 6.3 and $7.3 \AA$ ) form the molecular axis (Ref. 24). We take for $a_{Z}$ the average of these two values. In a more advanced model, using the formalism of Ref. 21, one can treat Z-DNA phosphates as four spirals of charges with different radii.

${ }^{23}$ The convergence of the series in (1) depends on how fast the argument of the Bessel functions grows with $n$. In Fig. 1 we have taken 30 terms in Eq. (1). Counting the next terms does not change the shape of $E_{Z}-E_{B}$ as a function of $n_{0}$ but just shifts the energy towards higher values.

${ }^{24}$ B. Pullman, R. Lavery, and A. Pullman, Eur. J. Biochem. 124, 229 (1982).

${ }^{25}$ J. B. Hasted, D. M. Ritson, and C. H. Collie, J. Chem. Phys. 16, 1 (1948).

${ }^{26}$ Equation (1) was obtained as the solution outside the spiral.

${ }^{27}$ J. H. van de Sande, L. P. McIntosh, and T. M. Jovin, EMBO J. 1, 777 (1982).

${ }^{28}$ M. Behe and G. Felsenfeld, Proc. Natl. Acad. Sci. U.S.A. 78, 1619 (1981).

${ }^{29}$ J. Granot et al., Biopolymers 21, 181 (1982); 21, 203 (1982) 21, 219 (1982) 21, 873 (1982).
${ }^{30}$ A. A. Kornyshev and S. Leikin, Phys. Rev. Lett. 82, 4138 (1999).

${ }^{31}$ A. G. Cherstvy, A. A. Kornyshev, and S. Leikin, J. Phys. Chem. B 106, 13362 (2002)

${ }^{32}$ A. G. Cherstvy, A. A. Kornyshev, and S. Leikin, J. Phys. Chem. B 108, 6508 (2004).

${ }^{33}$ A. G. Cherstvy, J. Phys.: Condens. Matter 17, 1363 (2005).

${ }^{34}$ For conditions favoring DNA condensation, the intermolecular interactions can also influence $B-Z$ transition. For instance, $\mathrm{Ni}(\mathrm{II}), \mathrm{Mn}(\mathrm{II})$, and $\mathrm{Zn}$ (II) bind strongly to guanine $N 7$ in the DNA major groove. This site is more accessible in $Z$ form that can trigger $B-Z$ transition in GC-rich DNA. A better charge alternation of DNA phosphates and adsorbed cations along the DNA axis is achieved when the latter are bound in the major groove. For juxtaposed parallel DNAs this results in a zipperlike DNA-DNA electrostatic attraction (Ref. 30) and DNA condensation. ${ }^{31-33}$ For AT-rich DNA these divalent cations can prefer to bind in the minor groove, with a lower electrostatic potential. That effectively diminishes the charge alternation along the DNA axis and thus the ability of such cations to condense AT-rich DNAs and to induce $B-Z$ transition in their solutions. 